\title{
Statistical analysis of short-wave fadeout for extreme space weather event estimation
}

\author{
Chihiro Tao $^{1 *} \mathbb{0}$, Michi Nishioka', Susumu Saito², Daikou Shiota', Kyoko Watanabe ${ }^{3}$, Naoto Nishizuka',
}

Takuya Tsugawa ${ }^{1}$ and Mamoru Ishii ${ }^{1}$

\begin{abstract}
Solar flares trigger an increase in plasma density in the ionosphere including the $\mathrm{D}$ region, and cause the absorption of radio waves, especially in high-frequency (HF) ranges, called short-wave fadeout (SWF). To evaluate the SWF duration and absorption statistically, we analyze long-term (36 years) ionosonde data observed by the National Institute of Information and Communications Technology (NICT). The minimum reflection frequency, fmin, is used to detect SWFs from 15-min-resolution ionosonde observations at Kokubunji, Tokyo, from 1981 to 2016. Since fmin varies with local time (LT) and season, we refer to $\mathrm{dfmin}$, which is defined as fmin subtracted by its 27-day running median at the same LT. We find that the occurrence of SWFs detected by three criteria, (i) $\mathrm{dfmin} \geq 2.5 \mathrm{MHz}$, (ii) $\mathrm{dfmin} \geq 3.5 \mathrm{MHz}$, and (iii) blackout, during daytime associated with any flare(s) greater than the C1 class is maximized at local noon and decreases with increasing solar zenith angle. We confirm that the dfmin and duration of SWFs increase with the solar flare class. We estimate the absorption intensity from observations, which is comparable to an empirical relationship obtained from sudden cosmic noise absorption. A generalized empirical relationship for absorption from long-distance circuits shows quantitatively different dependences on solar flare flux, solar zenith angle, and frequency caused by different signal passes compared with that obtained from cosmic noise absorption. From our analysis and the empirical relationships, we estimate the duration of extreme events with occurrence probabilities of once per 10, 100 , and 1000 years to be 1.8-3.6, 4.0-6.8, and 7.4-11.9 $\mathrm{h}$, respectively. The longest duration of SWFs of about $12 \mathrm{~h}$ is comparable to the solar flare duration derived from an empirical relationship between the solar flare duration and the solar active area for the largest solar active region observed so far.
\end{abstract}

Keywords: Short-wave fadeout (SWF), Dellinger effect, lonosphere, Solar flare, lonosonde, HF radio, Space weather

\section{Introduction}

Solar flares, one of the biggest explosive phenomena within the solar system, release emissions of various wavelengths from radio waves to gamma rays, and energetic particles over a few minutes to hours (e.g., Fletcher et al. 2011). Energetic-particle-driven solar radiation storms last for days. An increase in ionospheric plasma density up to the low-altitude $\mathrm{D}$ region owing to solar $\mathrm{X}$-ray emission causes the absorption of radio waves, especially in high-frequency (HF) ranges, which is

\footnotetext{
*Correspondence: chihiro.tao@nict.go.jp

${ }^{1}$ National Institute of Information and Communications Technology

(NICT), Tokyo, Japan

Full list of author information is available at the end of the article
}

called short-wave fadeout (SWF) or the Dellinger effect (e.g., Dellinger 1937). This SWF can interrupt transionospheric radio communication systems including ground-to-ground radio communication, satellite communication, and disaster prevention radio systems (e.g., US National Science and Technology Council 2018). In addition to SWFs, there are various sudden ionospheric disturbances (SIDs) associated with solar flares, as summarized by Davies (1996). Sudden cosmic noise absorption (SCNA) is also caused by plasma enhancement in the ionospheric $\mathrm{D}$ region. A sudden increase in total electron content (SITEC) is caused by an increase in plasma density in the $E$ and $F$ regions and has recently been well investigated by Global Navigation Satellite System (GNSS) monitoring methods. 
Ionospheric absorption is mainly measured by the following four methods (e.g., Mitra 1970): (a1) the A1 method based on vertical incident pulse reflection, (a2) the A2 method based on cosmic radio noise absorption using an instrument called a riometer (relative ionospheric opacity meter, extraterrestrial electromagnetic radiation), (a3) the $\mathrm{A} 3$ method based on an oblique ray path at frequencies over $2 \mathrm{MHz}$, and (a4) the minimum reflection frequency fmin from vertical incident ionograms. These methods have been widely used to monitor and evaluate the ionospheric response to solar flares.

During a sudden increase followed by a gradual decrease in solar X-ray flux associated with a solar flare, the signal absorption increases suddenly and sharply for a few minutes after the solar flare, then recovers over about half an hour (e.g., Dellinger, 1937; Chakraborty et al. 2018). An altitudinal variation in ionospheric density associated with SWFs was revealed by Digisonde observation (Handzo et al. 2014). Statistical analysis revealed that the occurrence of SWFs increases with solar activity during 11-year solar cycles (Hendl and Skrivanek 1973; Davies 1996). In an (a3) observation at Boulder, Colorado, the mean SWF duration of $\sim 10^{4}$ events over 1980-1987 was 23 min with $58.9 \%$ of them having a duration less than $14 \mathrm{~min}, 21.4 \%$ of them having a duration of $15-29 \mathrm{~min}, 4.3 \%$ having a duration of 30-44 $\mathrm{min}$, and about 3\% continuing for longer than 90 min (Davies, 1996). The duration decreases with increasing solar zenith angle, as observed by SuperDARN facilities at several observation stations for the same flare events (Chakraborty et al. 2018).

Sato (1975) proposed empirical relationships based on (a4) ionosonde and (a2) SCNA observations during solar flares greater than the $\mathrm{C} 1$ class $\left(=10^{-6} \mathrm{~W} / \mathrm{m}^{2}\right.$ at $1-8 \AA$ band) to estimate fmin and the absorption intensity $L$ as functions of the solar flux $F_{0}\left[\mathrm{~mW} / \mathrm{m}^{2}\right]$, solar zenith angle $\chi$ [rad $]$, and frequency $f[\mathrm{MHz}]$ as follows:

$$
\begin{aligned}
& f \min (\mathrm{MHz})=10 F_{0}^{1 / 4} \cos ^{1 / 2} \chi, \\
& L(\mathrm{~dB})=4.37 \times 10^{3} f^{-2} F_{0}^{1 / 2} \cos \chi .
\end{aligned}
$$

Equation (1) is based on observations from January 1972 to December 1973. Sato (1975) explained these dependences theoretically, referring to the "non-deviative" radio wave absorption in the low-altitude ionosphere under some assumptions including an ionospheric density profile with the Chapman formula. Maeda and Inuki (1972) proposed an empirical equation representing the degree of SWF based on the observed absorption intensity of long-distance short-wave circuits (a3).

Barta et al. (2019) reported that simultaneous observations using several ionosonde observation facilities located along the meridional longitude from low to middle latitudes showed the dependence of fmin on solar flare flux and solar zenith angle. They defined $\mathrm{d} f \mathrm{~min}$ as the difference between the values of $\mathrm{fmin}$ and the mean fmin for reference days. They suggested that $\mathrm{d} f \mathrm{~min}$ is a good qualitative measure for the relative variation in non-deviative absorption intensity, especially in the case of less intense solar flares, which do not cause total radio fadeout in the ionosphere (<M6-class).

How large extreme space weather events could occur and how probable such events are-these are important questions for both scientific interest and the protection of social infrastructure. Riley (2012) estimated the probability of occurrence of solar flare flux, the speed of coronal mass ejection, the Dst index representing magnetospheric storms, and extreme proton events. To evaluate the size of extreme events, he used the complementary cumulative distribution function, defined as the probability of an event with a magnitude greater than or equal to a certain critical value. Nishioka et al. (submitted to Earth Planets and Space) applied the method to ionospheric total electron contents based on long-term observations using GNSS and ionosondes. However, the probability of occurrence of extreme SWF events has not yet been investigated.

SWF is a space-weather phenomenon having an adverse effect on modern civilization and technologies. For the design and operation of radio communication systems, it is important to know how long an SWF event will last to predict how soon the operation of systems will recover to normal. The expected SWF duration is also important information to prepare alternative means of communication during the event.

In this study, we analyze long-term (36 years) ionospheric sounder (ionosonde) data observed by the National Institute of Information and Communications Technology (NICT) to evaluate the SWF duration with modified $\mathrm{d} f$ min criteria. We focus on the duration and absorption intensity to estimate extreme SWF events. The duration is based on the results of our statistical analysis. For the absorption intensity, we refer to empirical relationships proposed by Sato (1975), i.e., Eqs. (1) and (2), and those proposed by Maeda and Inuki (1972) based on long circuit observations (a3). Since the equations of Maeda and Inuki (1972) refer to three wavelength bands of solar X-ray flux, we generalize them to obtain a simple relationship between the absorption intensity and the solar flare flux at 1-8 $\AA$. We also examine the applicability of these equations based on a specific period (<2 years) by comparison with our observations. Next section describes the ionosonde observations and the data set used in this study followed by sections reporting 
the statistical analysis and results related to the SWF duration and the absorption intensity.

\section{lonosonde observations and data set}

Four ionosonde facilities are currently continuously operated in Japan by NICT. We use one of them, Kokubunji station $\left(35.71^{\circ} \mathrm{N}\right.$ latitude, $139.49^{\circ} \mathrm{E}$ longitude), in this study. SWF phenomena require high-time-resolution observation. Manually scaled parameters with 15-min resolution have been available for Kokubunji station since 1981. The manually scaled parameters of other stations are usually available at a 1-h cadence. In 2017, the ionosonde facility at Kokubunji was updated to Vertical Incidence Pulsed Ionospheric Radar 2 (VIPIR2) instruments, which can record calibrated signal intensity. The VIPIR2 system was occasionally operated in 2016 and enables us to evaluate signal attenuation by ionospheric absorption as described in "Absorption observed by VIPIR2" section.

The ionosonde at Kokubunji transmits HF radio pulses vertically from 1 to $30 \mathrm{MHz}$ within 15 or $30 \mathrm{~s}$ and receives reflected signals at $15 \mathrm{~min}$ intervals. The ionospheric height is calculated from the traveling time of the sounding radio wave multiplied by the light velocity and is called the virtual height. The observed ionogram contains several important features including the minimum reflection frequency fmin used in this study. When the reflected echo is not observed, a flag " $\mathrm{B}$ ", meaning blackout, is set instead of $f$ min.

For the solar flare information, the Geostationary Operational Environmental Satellite (GOES) flare list provided by National Oceanic and Atmospheric Administration (NOAA) National Centers for Environmental Information (https://www.ngdc.noaa.gov/stp/space -weather/solar-data/solar-features/solar-flares/x-rays/ goes/xrs/) over the period 1975-mid-2017 is used in this study. We analyze the 1981-2016 data set.

\section{SWF duration}

\section{Example of observed event}

Figure 1 shows an example of ionosonde observation during a solar flare event on November 10, 2004. A solar X2.5-class flare began at local time $(\mathrm{LT}=\mathrm{UT}+9 \mathrm{~h})$ 10:59 and reached a peak at 11:20 (Fig. 1a). The ionogram at 11:00 (Fig. 1d, upper left) shows a weak echo signal at about $2 \mathrm{MHz}$ at an altitude of $100-150 \mathrm{~km}$ (red circle) and a strong echo signal at $4-9 \mathrm{MHz}$ at $220-450 \mathrm{~km}$. We can also easily find vertical lines corresponding to artificial signals. $f$ min is $2.1 \mathrm{MHz}$ at this timing. The ionogram at $11: 15$, around the flare peak time, shows the disappearance of the echo signal and most of the artificial signals. The echo signal gradually recovered, starting from a higher frequency at a higher altitude, e.g., the echo signal is seen at $>5.5 \mathrm{MHz}$ and $>275 \mathrm{~km}$ at $11: 30$, then at $>4.0 \mathrm{MHz}$ and $>250 \mathrm{~km}$ at 12:00. The recovery of the low-altitude echo is observed at 12:15 (red circle at the right top of Fig. 1d). The corresponding fmin values are $2.1 \mathrm{MHz}$, 'B', 5.4 MHz, 4.3 MHz, 4.0 MHz, and 2.6 MHz for 11:00-12:15 (Fig. 1b). Since the echo disappears from the low frequency and low altitude, where there is greater absorption, the $f$ min variation is an indicator of SWF events.

Note that $f$ min varies with the season, local time, and solar activity. Figure 2a shows seasonal variations in monthly averaged fmin values of four groups of solar minima and solar maxima. fmin increases from April to September and is higher than that during the northern winter, reflecting the variation in solar zenith angle in Japan, with a moderate increase $(\sim 0.5 \mathrm{MHz})$ for periods with high solar activity (1989-1991 and 2000-2002). The increase during the solar maximum around 2011 was not significant $(\sim 0.1 \mathrm{MHz})$, which was due to the weaker solar activity of the solar cycle. fmin increases during daytime with the highest value at LT 11:00-12:00 (Fig. 2c). The dependence of $f \mathrm{~min}$ on local time is much stronger, with an amplitude of $\sim 1.5 \mathrm{MHz}$ for the high solar activities around 1992 and 2003 and an amplitude of only $\sim 0.3 \mathrm{MHz}$ for the minimum solar activities in 2008 and around 2016.

To quantitatively measure the short-time variation in fmin owing to a solar flare beyond these LT and solar activity dependences, we refer to $\mathrm{d}$ fmin, which is defined in this study as fmin subtracted by its 27-day running median at the same LT. The $\mathrm{d}$ fmin values of the event in Fig. 1 are almost zero $(0.0 \mathrm{MHz})$ before the event $(-11: 00)$, increase to $3.6 \mathrm{MHz}$ at 11:30, and decrease to $2.5 \mathrm{MHz}$ at 11:45 (Fig. 1c).

\section{fmin and dfmin}

Firstly, we examine the maximum fmin and $\mathrm{d}$ fmin values within $1 \mathrm{~h}$ of the occurrence of a solar flare of and above the $\mathrm{C} 1$ class $\left(10^{-6} \mathrm{~W} / \mathrm{m}^{2}\right)$. The average $f$ min value is $2.1 \mathrm{MHz}$ at the C1-class flare level, then it gradually increases with solar X-ray flux up to $3.5 \mathrm{MHz}$ at the X1-class flare level (Fig. 3a). $\mathrm{d}$ fmin also shows a similar trend to solar X-ray flux up to $0.32 \mathrm{MHz}$ at the C1-class flare level and $1.8 \mathrm{MHz}$ at the X1-class flare level (Fig. 3d). The decreasing trend seen in both fmin and $\mathrm{d}$ fmin above the X2-class flare level might be caused by the small number of events (blue line) with dependence on the solar zenith angle (see the next paragraph). The standard deviation (shown by error bars) also increases for larger flares.

The solid and dashed red curves in Fig. 3a are fmin values derived from the empirical Eq. (1) (Sato 1975) for subsolar point (solar zenith angle $X=0^{\circ}$ ) and $X=88^{\circ}$ cases, respectively. Almost all the obtained values are 

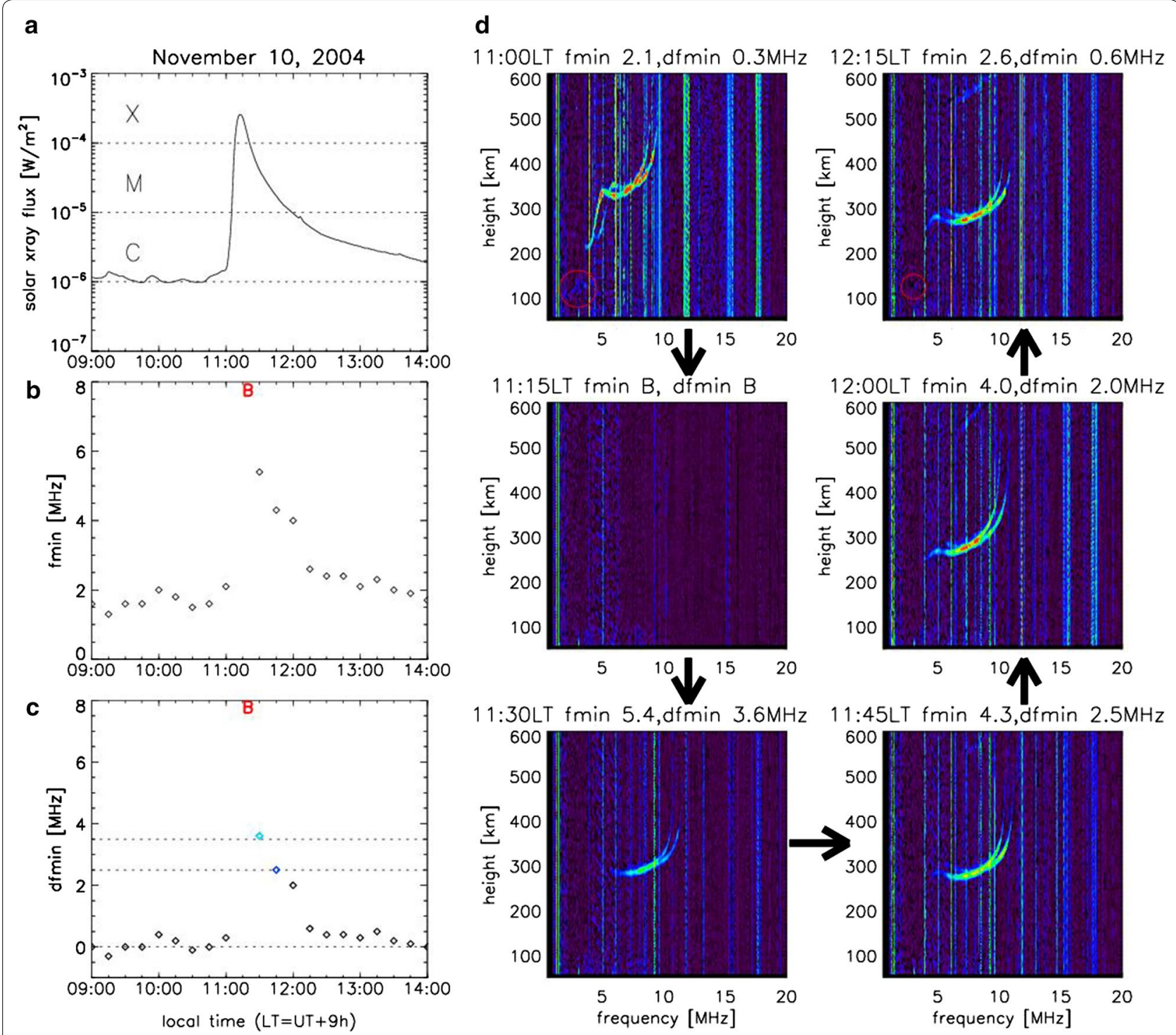

Fig. 1 Time variations in a solar X-ray flux, b fmin, c dfmin, and d ionograms during solar flare events on November 10, 2004. The fmin and $\mathrm{dfmin}$ values of the blackout period are shown as "B" in red in b, c. dfmin $\geq 2.5 \mathrm{MHz}$ and dfmin $\geq 3.5 \mathrm{MHz}$ values are shown in blue and light blue, respectively, in c. The weak echo signal at $\sim 3 \mathrm{MHz}$ at altitudes of 100-150 km is surrounded by red circles in d at LT 11:00 and 12:15

within the two curves. Figure 3b, e shows the scatter plots of fmin and $\mathrm{d}$ fmin, respectively, as a function of $\cos X$ and solar X-ray flux. Equation (1) with $f \min =2.5 \mathrm{MHz}$ (blue line), $3.5 \mathrm{MHz}$ (light blue), and $5 \mathrm{MHz}$ (green) represents the variations in $f$ min and $d$ fmin well. Figure $3 c$, f, respectively, shows the fmin and $\mathrm{d}$ fmin values normalized by $(\cos x)^{0.5}$. The trend of a continuous increase beyond the $\mathrm{X} 2$-class is clearly seen.

The rate of increase in normalized fmin is represented well by the relationship proposed by Sato (1975), although the values are slightly larger than those obtained on the basis of the relationship. This is considered to be caused by the long-term fmin variation seen in Fig. 2. Assuming the same dependence of $\mathrm{d} f$ min on the solar flux $\left(\propto F_{0}^{1 / 4}\right)$ and solar zenith angle $\left(\propto \cos ^{1 / 2} \chi\right)$, because they are explained by theoretical analysis (Sato, 1975), the $\chi^{2}$ fitting provides the following relationship:

$$
\mathrm{d} f \min / \cos ^{1 / 2} \chi=8.7 F_{0}^{1 / 4}-1.35,
$$

where the $95 \%$ confidence level corresponds to the coefficients of $8.7 \pm 1.6$ and $1.35 \pm 0.45$. The blue line in Fig. $3 f$ shows this equation. 


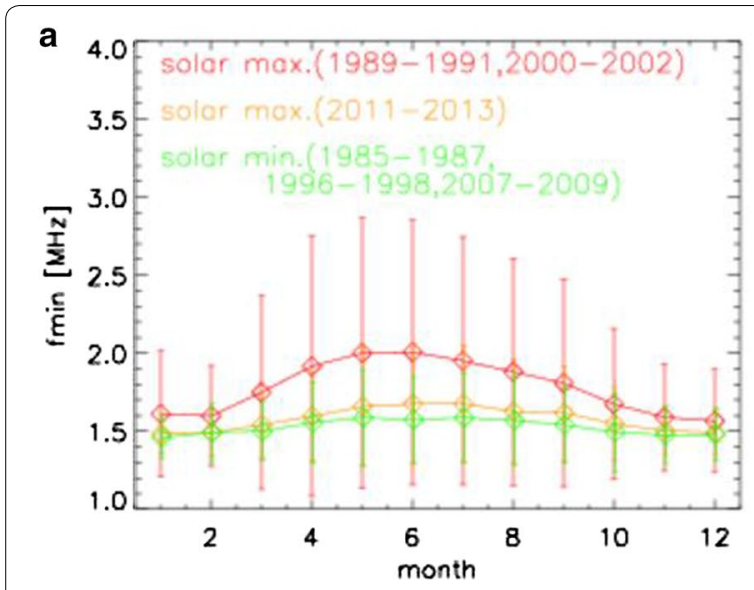

b

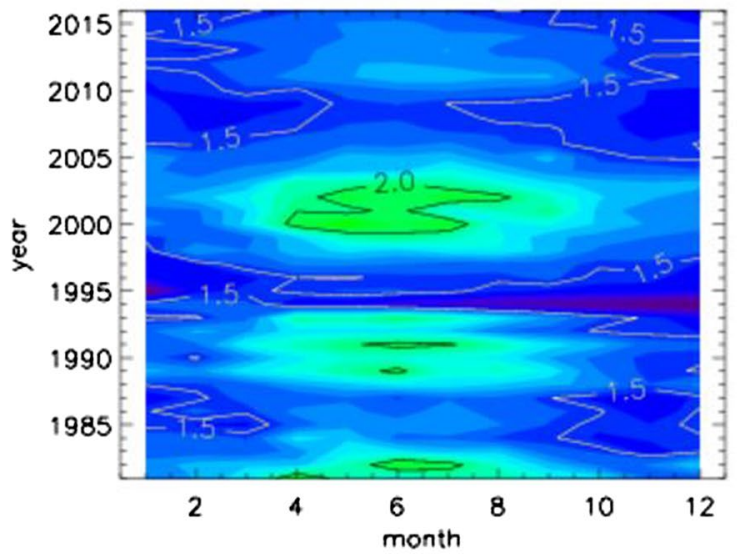

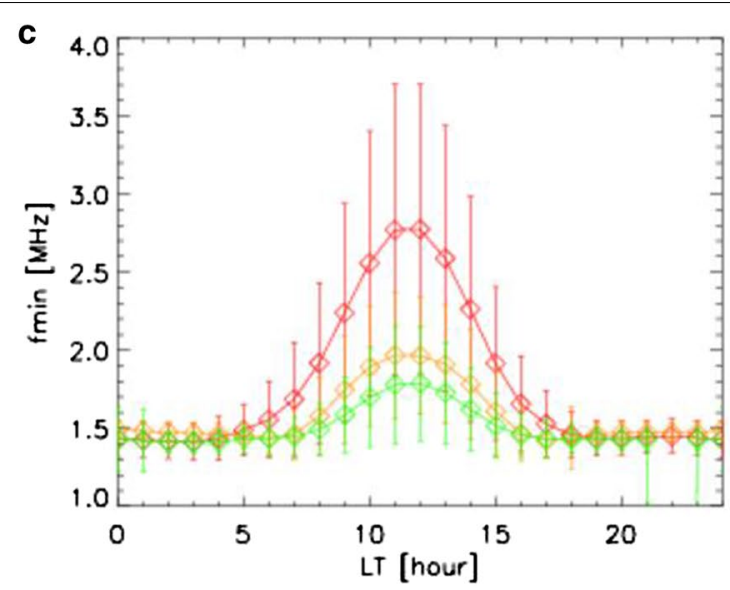

d

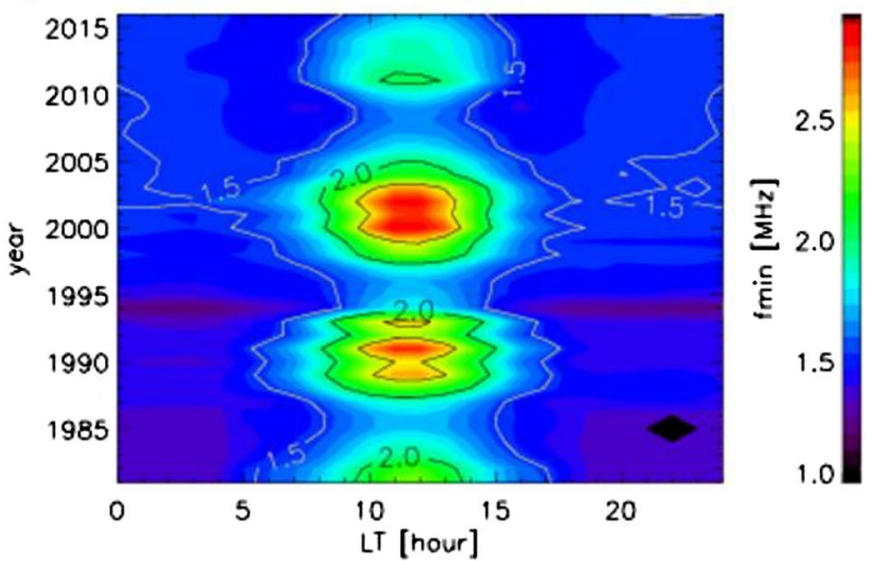

Fig. 2 a Seasonal variation in monthly averaged fmin as a function of month, b contour map of monthly averaged fmin values in MHz as a function of month and year, c averaged fmin as a function of $L T(=U T+9 \mathrm{~h}$ ), and $\mathrm{d}$ contour map of fmin values in $\mathrm{MHz}$ as a function of $L T$ and year. Vertical lines in $\mathrm{a}, \mathrm{c}$ show the standard deviation, \pm 10 , and line colors distinguish the solar minima and two groups of solar maxima, as labeled in a

Equation (1) is based on the observation from January 1972 to December 1973, which corresponds to a declining phase of solar activity. The number of solar sunspots in 1972-1973 was comparable to that around 2012 at the solar rising phase in cycle 24 . The daytime $f \min$ in 2012 is about $2 \mathrm{MHz}$ according to Fig. 2c. The difference between Eq. (1) shown by the red line in Fig. 3f and Eq. (3) shown by the blue line in Fig. $3 \mathrm{f}$ is also about $2 \mathrm{MHz}$. The difference between the statistically normalized $f$ min variation and the red line in Fig. $3 \mathrm{c}$ is considered to be caused by the difference in the background $f \mathrm{~min}$, i.e., the long-term variation of $f \mathrm{~min}$, when the data used were obtained. The standard deviation of the difference between $\mathrm{d}$ fmin from Eq. (3) and the observed values is evaluated as $0.62 \mathrm{MHz}$.

\section{Event selection for duration analysis}

To focus on the fmin variation and the occurrence of blackout relevant to solar flares, we exclude events occurring during the night in Japan (after LT 19:00 and before LT 05:00) and those not associated with a solar flare above the $\mathrm{C} 1$ class, i.e., peak $\mathrm{X}$-ray flux $\geq 10^{-6} \mathrm{~W} /$ $\mathrm{m}^{2}$, within $1 \mathrm{~h}$ of the occurrence of the solar flare. In addition to the solar flare list mentioned in "Ionosonde observations and data set" section, we also refer to the time variation of solar X-ray flux over the 1-8 $\AA$ band and exclude events with insufficient data. We categorize SWFs with the criteria of (i) $\mathrm{d} f \mathrm{~min} \geq 2.5 \mathrm{MHz}$ including blackout, (ii) $\mathrm{d} f \mathrm{~min} \geq 3.5 \mathrm{MHz}$ including blackout, and (iii) blackout (Table 1), and obtain the duration automatically. The obtained results are manually validated. According to Eq. (3), the events with $\mathrm{d}$ fmin of 2.5 and 3.5 at the sub-solar point approximately correspond to the flare sizes of M4 and X1, respectively. The occurrences of $\mathrm{d} f \min \geq 2.5 \mathrm{MHz}, \mathrm{d} f \mathrm{~min} \geq 3.5 \mathrm{MHz}$, and "B" over the entire interval were $0.15 \%, 0.068 \%$, and $0.027 \%$, respectively.

Here we briefly mention the limitation of this observation and analysis. We analyze SWFs associated with solar flare events referring to 1-8 $\AA$ flux. Note that Deshpande et al. (1972) reported that $12 \%$ of all SIDs occurred when 

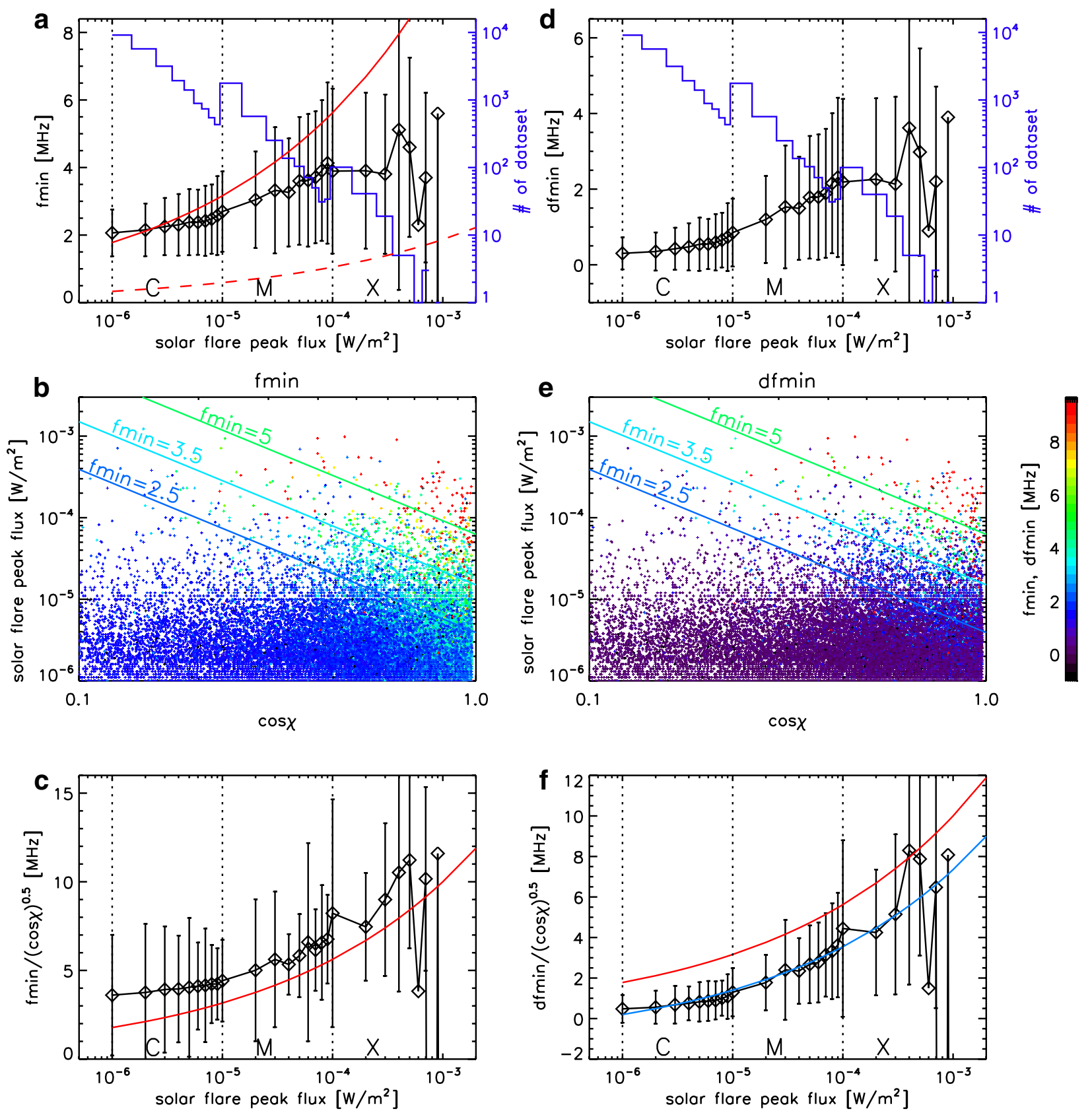

Fig. 3 Maximum a fmin and $d \mathrm{~d}$ fmin values within $\pm 1 \mathrm{~h}$ of solar flare as a function of solar flare peak flux, $\mathrm{b}$ fmin and e dfmin values as a function of solar flare peak flux and $\cos x$, where $x$ is the solar zenith angle, and $\mathrm{c} f \mathrm{~min}$ and $\mathrm{f} \mathrm{dfmin}$ normalized by $(\cos \mathrm{x})^{0.5}$ as a function of solar flare peak flux. The total number of events for this analysis is shown by a histogram with blue lines using the right $y$-axis scale in Fig. a, d. fmin and dfmin values respectively in $b$, e are distinguished by the same color from the color bar on the right side, and blue, light blue, and green lines show Eq. (1) for $\mathrm{fmin}=2.5,3.5$, and $5 \mathrm{MHz}$, respectively. Vertical solid lines in $\mathrm{a}, \mathrm{c}, \mathrm{d}$, and $\mathrm{f}$ show the standard deviation, $\pm 1 \sigma$. In $\mathrm{a}$, the red curve shows Eq. (1) at the subsolar point $\left(X=0^{\circ}\right)$ and the dashed red line shows Eq. (1) at $X=88^{\circ}$. Solid red curves in $\mathrm{c}$ and $\mathrm{f}$ show the normalized Eq. (1), and the blue curve in $\mathrm{f}$ shows best-fitted function represented by Eq. (3)

the solar 0-8 $\AA$ peak flux was less than $10^{-6} \mathrm{~W} / \mathrm{m}^{2}$, i.e., C1-class, and half of them (7\% of all SIDs) were associated with the hardening of the solar flux spectrum. Since the time resolution of our observation is usually $15 \mathrm{~min}$, we cannot evaluate the exact duration beyond this interval. If we observe SWF events at one, two, three, ... data 
Table 1 Event detection criteria for SWF duration analysis

\begin{tabular}{lll}
\hline Criteria (\#) & \multicolumn{2}{l}{ Event detection criteria } \\
\cline { 2 - 3 } & dfmin & Additional \\
\hline (i) & $\geq 2.5 \mathrm{MHz}$ & Day time LT (05 h-19 h), flare $\geq \mathrm{Cl}$ class \\
(ii) & $\geq 3.5 \mathrm{MHz}$ & \\
(iii) & "B"(Black out) & \\
\end{tabular}

points, then we simply count them as events with 15,30 , $45, \ldots$ min duration, respectively. Note that, for example, the actual event duration for a two-data-point observation is within 15-45 min. This time resolution is coarser than that of other SWF observations, e.g., SuperDARN observation (Nishitani et al. 2019). We discuss the effect of this time resolution on the result in Sect. 3.4.

\section{Results for event duration}

From the analysis using the data set over 1981-2016, we detected 616, 302, and 120 events for the criteria (i) $\mathrm{d} f \mathrm{~min} \geq 2.5 \mathrm{MHz}$ including blackout, (ii) $\mathrm{d} f \min \geq 3.5 \mathrm{MHz}$ including blackout, and (iii) blackout, respectively.

Figure 4a shows a histogram of the SWF duration. All the criteria (i)-(iii) show a decreasing SWF number with increasing SWF duration. Figure 4b shows the occurrence ratio, i.e., the event number divided by the total number, of SWF events with different criteria. From this analysis with criterion (i), we found that $79 \%$ of the events have one or two continuous 15-min-resolution timings, $11 \%$ have $4-7$ continuous timings, and $4.2 \%$ have 8 or more continuous timings. These ratios are $78 \%, 11 \%$, and $3.6 \%$ for criterion (ii) and $78 \%, 14 \%$, and $2.5 \%$ for criterion (iii), respectively. The distribution profiles are similar among the different SWF levels.

This is in agreement with the previous study based on observations at Boulder, Colorado, with high time resolution by Davies (1996): $80.3 \%$ of the events have a duration $<30 \mathrm{~min}, 12.2 \%$ have a duration of 60-119 min, and $1.1 \%$ have a duration $\geq 120 \mathrm{~min}$. Although the time resolution of our data set, $15 \mathrm{~min}$, is coarse, we confirm that observations with high time resolution provide a similar distribution of event duration for a long time scale.
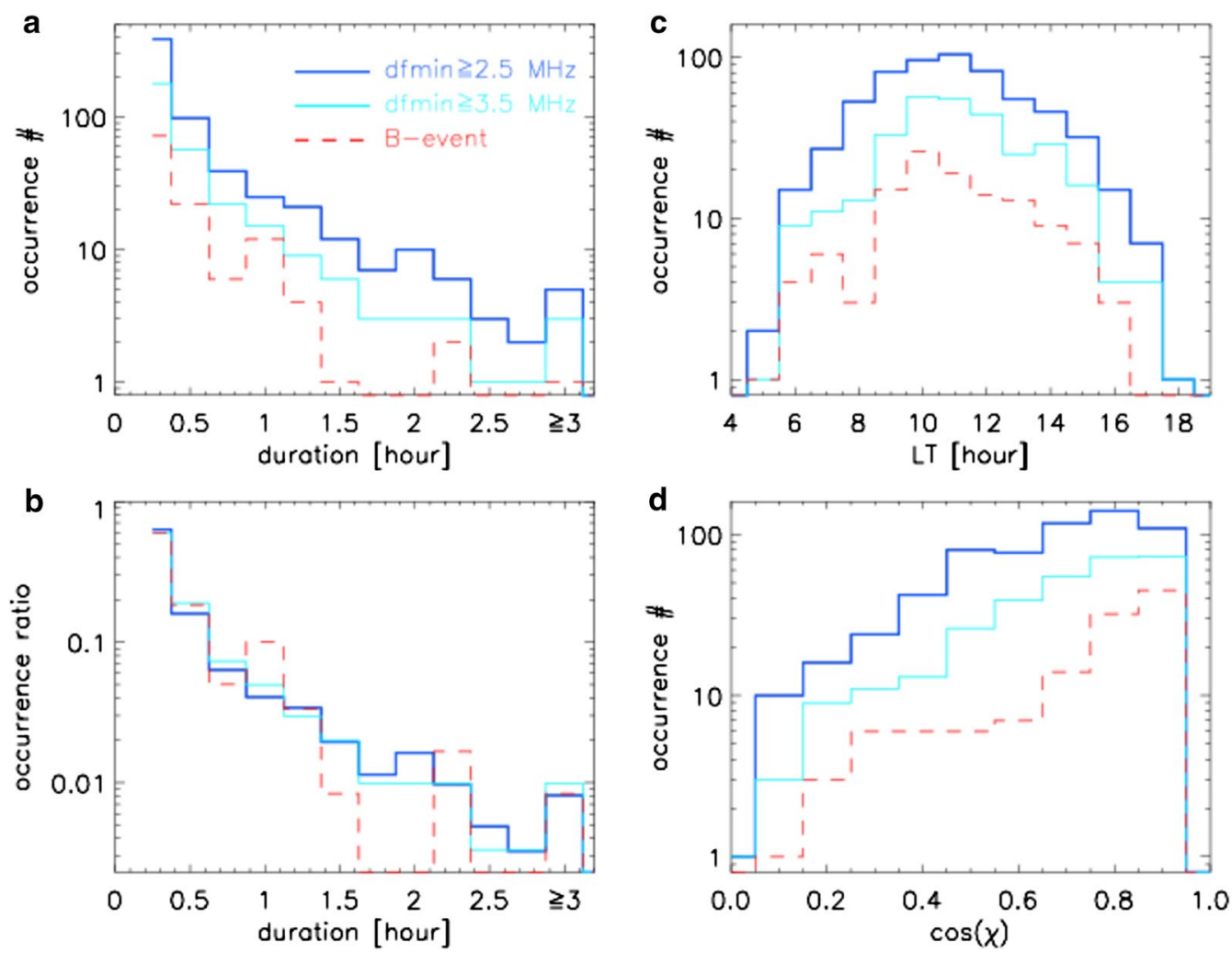

Fig. 4 Histograms of a SWF event number and b occurrence ratio as a function of SWF event duration, and histograms of SWF event number as a function of $C L T(=U T+9 h$ ) and d solar zenith angle for SWF events with the criteria of $\mathrm{dfmin} \geq 2.5 \mathrm{MHz}$ (blue lines), dfmin $\geq 3.5 \mathrm{MHz}$ (light blue lines), and blackout (red dashed lines) 
Figure 4c, d shows the dependence of events of criteria (i)-(iii) on local time and solar zenith angle, respectively. As indicated, more events are obtained at noon and with a larger $\cos (\chi)$.

The event with the longest duration of $8 \mathrm{~h} 15 \mathrm{~min}$ for criterion (i) occurred on April 3, 2001 with at least two large solar flares: X17 and X1.2. The event with the longest duration of $5 \mathrm{~h} 15 \mathrm{~min}$ for criterion (ii) was also related to the X17 flare on April 3, 2001. The event of $3.25 \mathrm{~h}$ for criterion (iii) was associated with the X1.0 solar flare on August 15, 1989.

The temporal variations in solar flux, fmin, $\mathrm{d} f \mathrm{~min}$, and ionosonde signal during the longest SWF event, which occurred on April 3, 2001, are shown in Fig. 5. The solar $\mathrm{X}$-ray flux increases several times within the day, with the largest increase for X17 at LT 7:03 followed by that for $\mathrm{X} 1.2$ at 12:55 (Fig. 5a). The temporal variation in ionosonde echo power as a function of frequency is represented by the largest signal in the range of $60-500 \mathrm{~km}$ at each frequency (Fig. 5d). The broadband red area, which was seen at $1-10 \mathrm{MHz}$ at night and shifted up to $12 \mathrm{MHz}$ during daytime, represents the ionospheric echo with its minimum frequency corresponding to $\mathrm{fmin}$, as shown by black pluses. The horizontal thin lines, e.g., at 10 and $12 \mathrm{MHz}$, are artificial noise. Around these flare peaks at $\sim$ 7:00 and 13:00, echo signals were lost (blackout, blue part in Fig. $5 \mathrm{~d}$ ) as fmin and dfmin became "B" (Fig. 5b, c). After the X17 flare, the signal at $8-12 \mathrm{MHz}$ appeared with artificial noise, while maintaining large fmin values. After the X1.2 flare, the signal recovered with decreasing $f$ min and $\mathrm{d} f \mathrm{~min}$ by $16: 45$.

Figure 6a shows the correlation of the SWF duration with the flare peak flux for all events. The correlation, however, is not significant according to the results of an analysis of variance (ANOVA). Figure 6b, c, respectively, shows the same plot for cases with solar zenith angles of $0-45^{\circ}$ and $45-90^{\circ}$. The same duration tends to be associated with a larger flare class for the cases with larger solar zenith angles, as expected. A significant correlation between SWF duration $(\leq 1.5 \mathrm{~h})$ and flare size is detected by the ANOVA test for the solar zenith angles of $0-45^{\circ}$.

\section{Extreme event estimation for SWF duration}

From the results of the analysis in "Results for event duration" section, we obtain the complementary cumulative distribution function (Fig. 7). This counts the number of events larger than the value shown in the $x$-axis. We choose a quadratic function rather than a linear function for the extrapolation to avoid overestimation. In addition, quadratic functions fit the observed distribution better than linear functions. Using the functions derived from fittings for up to a $3 \mathrm{~h}$ duration, we estimate the duration for extreme events with occurrence probabilities of once per 1, 10, 100, and 1000 years, as summarized in Table 2. In the complete blackout case, the durations are $38 \mathrm{~min}, 1.8 \mathrm{~h}, 4.0 \mathrm{~h}$, and $7.4 \mathrm{~h}$, respectively. In the once per 1000 years case, the duration becomes $11.9 \mathrm{~h}$ for $\mathrm{d} f \mathrm{~min} \geq 2.5 \mathrm{MHz}$ and $11.5 \mathrm{~h}$ for $\mathrm{d} f \mathrm{~min} \geq 3.5 \mathrm{MHz}$. The extreme points, $8 \mathrm{~h} 15 \mathrm{~min}$ for $\mathrm{d} f \mathrm{~min} \geq 2.5 \mathrm{MHz}$ and $5 \mathrm{~h}$ $15 \mathrm{~min}$ for $\mathrm{d} f \mathrm{~min} \geq 3.5 \mathrm{MHz}$, are associated with continuous flares X17 and X1.2, which occurred within $6 \mathrm{~h}$ with gradual decay on April 3, 2001, as shown in Fig. 5. This suggests that frequent explosions of long-duration flares provide long-term SWFs. It is reported that a typical duration of compound X-class flare-driven SWF events can be much longer than that of events driven by isolated X-class flares, which is suggested to be the result of an extended ionospheric relaxation time due to a slow recovery of D-region electron temperature after large perturbations (Chakraborty et al. 2019 and references therein).

SWF duration is mainly determined by solar flare duration. The correlation between solar flare duration and flare ribbon area has been reported by several research groups. Flare ribbon is an emission due to collision between the solar chromosphere and energetic particles generated by solar flares. Reep and Knizhnik (2019) analyzed 2956 sets of solar flares and flare ribbons observed from April 2000 to April 2006 and derived the relationship between the full width at half-maximum (FWHM) time of the GOES X-ray variation $t[\mathrm{~s}]$, i.e., flare duration time, associated with an X-class flare and the ribbon area $A\left[\mathrm{~cm}^{-2}\right]$ observed at $160 \mathrm{~nm}$ wavelength as

$$
t=1.7 \times 10^{-25} A^{1.44}
$$

They found a low correlation for flares in small classes. Since SWFs are usually associated with middle M-class flares or higher, this FWHM time is expected to be a good indicator of the SWF duration. If we set the ribbon area to have the length of the solar radius and a width of $1 / 10$ of the solar radius, then the duration is estimated to be 1.2 days for the area of $4.9 \times 10^{20} \mathrm{~cm}^{2}$. This area is on the same order as the maximum size of solar spots in observational records of about $6000 \mathrm{MSH}=3.6 \times 10^{20} \mathrm{~cm}^{2}$ at AR 14886 observed by Royal Greenwich Observatory in April 1947 (e.g., Aulanier et al. 2013). This duration is considerably longer than the SWF duration of up to about $12 \mathrm{~h}$ proposed in this study. Since SWFs occur only during the daytime, their duration depends on the timing of the event initiation. For middle-latitude regions such as Japan, the longest duration is about $12 \mathrm{~h}$.

\section{SWF absorption intensity Absorption observed by VIPIR2}

As introduced in "Ionosonde observations and data set" section, the ionosonde VIPIR2 system was operated 

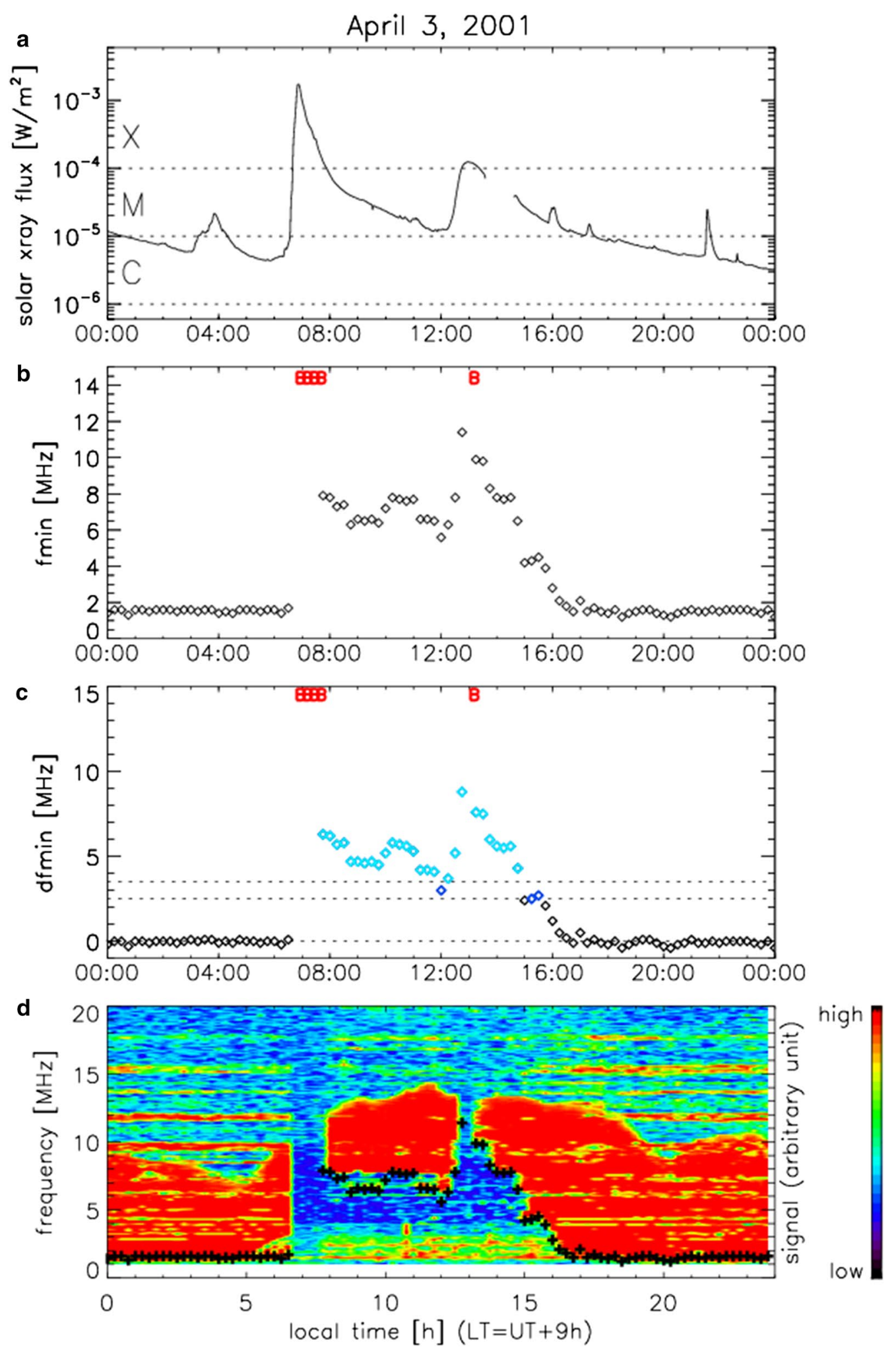

Fig. 5 Time variations in a solar X-ray flux, b fmin, $\mathrm{c} \mathrm{dfmin,} \mathrm{and} \mathrm{d} \mathrm{maximum} \mathrm{ionosonde} \mathrm{signal} \mathrm{at} \mathrm{each} \mathrm{frequency} \mathrm{during} \mathrm{the} \mathrm{longest} \mathrm{SWF} \mathrm{event}$

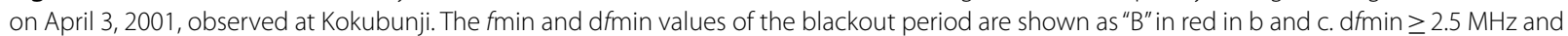
$\mathrm{dfmin} \geq 3.5 \mathrm{MHz}$ values are shown in blue and light blue, respectively, in c. Black pluses in d show fmin values. This event was recorded by the ionosonde system with an intensity resolution of 1 bit. The smoothing procedure in the analysis provides an arbitrary color scale with increasing signal intensity from blue to red 

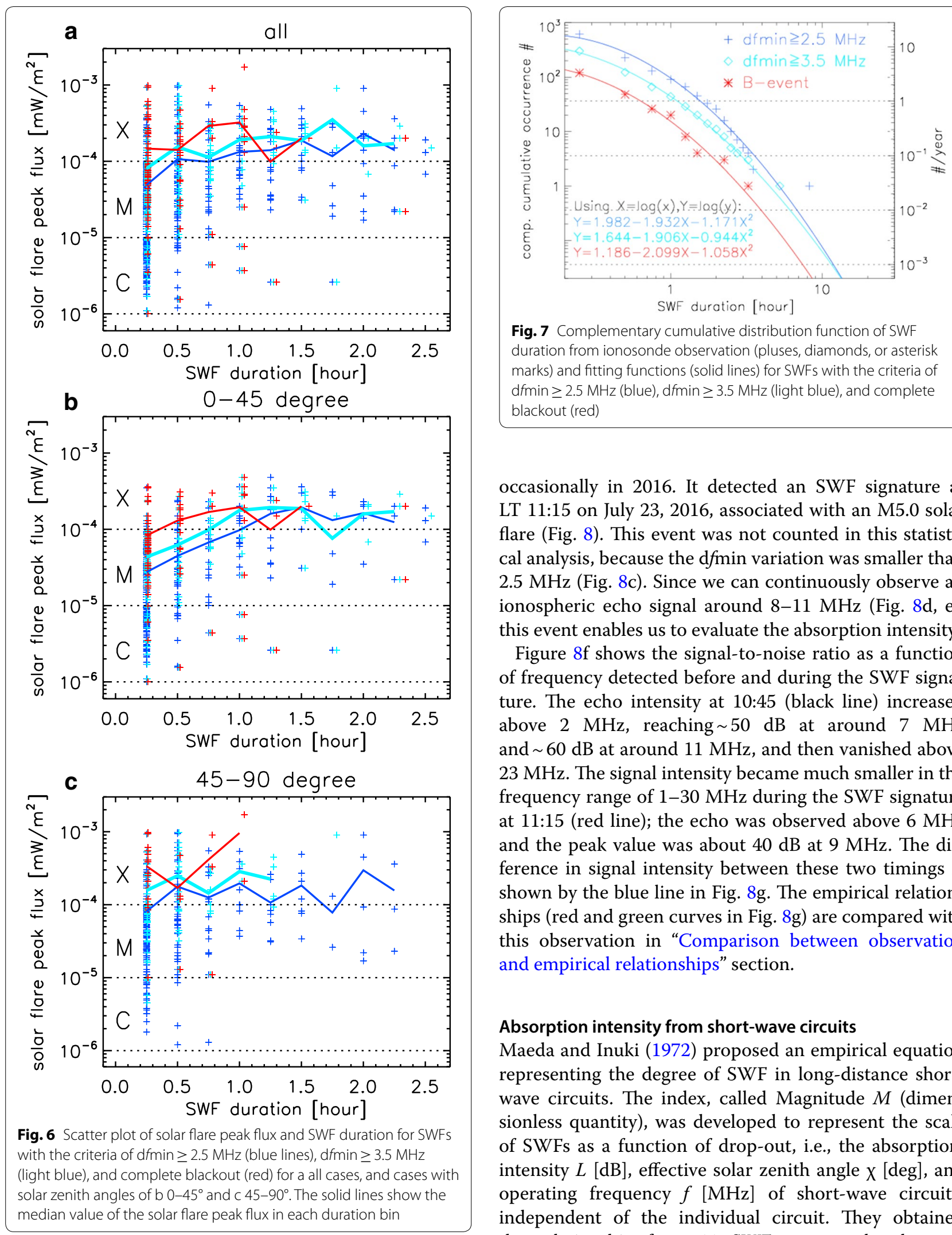

Fig. 7 Complementary cumulative distribution function of SWF duration from ionosonde observation (pluses, diamonds, or asterisk marks) and fitting functions (solid lines) for SWFs with the criteria of $\mathrm{d} f \mathrm{~min} \geq 2.5 \mathrm{MHz}$ (blue), $\mathrm{d} f \mathrm{~min} \geq 3.5 \mathrm{MHz}$ (light blue), and complete blackout (red)

occasionally in 2016. It detected an SWF signature at LT $11: 15$ on July 23,2016 , associated with an M5.0 solar flare (Fig. 8). This event was not counted in this statistical analysis, because the $\mathrm{d} f$ min variation was smaller than $2.5 \mathrm{MHz}$ (Fig. 8c). Since we can continuously observe an ionospheric echo signal around 8-11 MHz (Fig. 8d, e), this event enables us to evaluate the absorption intensity.

Figure $8 \mathrm{f}$ shows the signal-to-noise ratio as a function of frequency detected before and during the SWF signature. The echo intensity at 10:45 (black line) increased above $2 \mathrm{MHz}$, reaching $\sim 50 \mathrm{~dB}$ at around $7 \mathrm{MHz}$ and $\sim 60 \mathrm{~dB}$ at around $11 \mathrm{MHz}$, and then vanished above $23 \mathrm{MHz}$. The signal intensity became much smaller in the frequency range of $1-30 \mathrm{MHz}$ during the SWF signature at 11:15 (red line); the echo was observed above $6 \mathrm{MHz}$ and the peak value was about $40 \mathrm{~dB}$ at $9 \mathrm{MHz}$. The difference in signal intensity between these two timings is shown by the blue line in Fig. 8g. The empirical relationships (red and green curves in Fig. 8g) are compared with this observation in "Comparison between observation and empirical relationships" section.

\section{Absorption intensity from short-wave circuits}

Maeda and Inuki (1972) proposed an empirical equation representing the degree of SWF in long-distance shortwave circuits. The index, called Magnitude $M$ (dimensionless quantity), was developed to represent the scale of SWFs as a function of drop-out, i.e., the absorption, intensity $L[\mathrm{~dB}]$, effective solar zenith angle $\mathrm{X}[\mathrm{deg}]$, and operating frequency $f[\mathrm{MHz}]$ of short-wave circuits, independent of the individual circuit. They obtained the relationship from 11 SWFs commonly observed in the circuits of Hiraiso $\left(34.62^{\circ} \mathrm{N}\right.$ latitude, $135.05^{\circ} \mathrm{E}$ 
Table 2 Estimated duration for extreme events

\begin{tabular}{llllll}
\hline Duration of event $(\mathbf{h})$ & Once per year & Once per $\mathbf{1 0}$ years & Once per $\mathbf{1 0 0}$ years & Once per $\mathbf{1 0 0 0}$ years & Reference \\
\hline $\mathrm{d} f \min \geq 2.5 \mathrm{MHz}$ & 1.6 & 3.6 & 6.8 & 11.9 & Figure 7 ionosonde \\
$\mathrm{d} f \mathrm{~min} \geq 3.5 \mathrm{MHz}$ & 1.1 & 2.9 & 6.1 & 11.5 & Figure 7 ionosonde \\
"B"-event & 0.63 & 1.8 & 4.0 & 7.4 & Figure 7 ionosonde \\
\hline
\end{tabular}
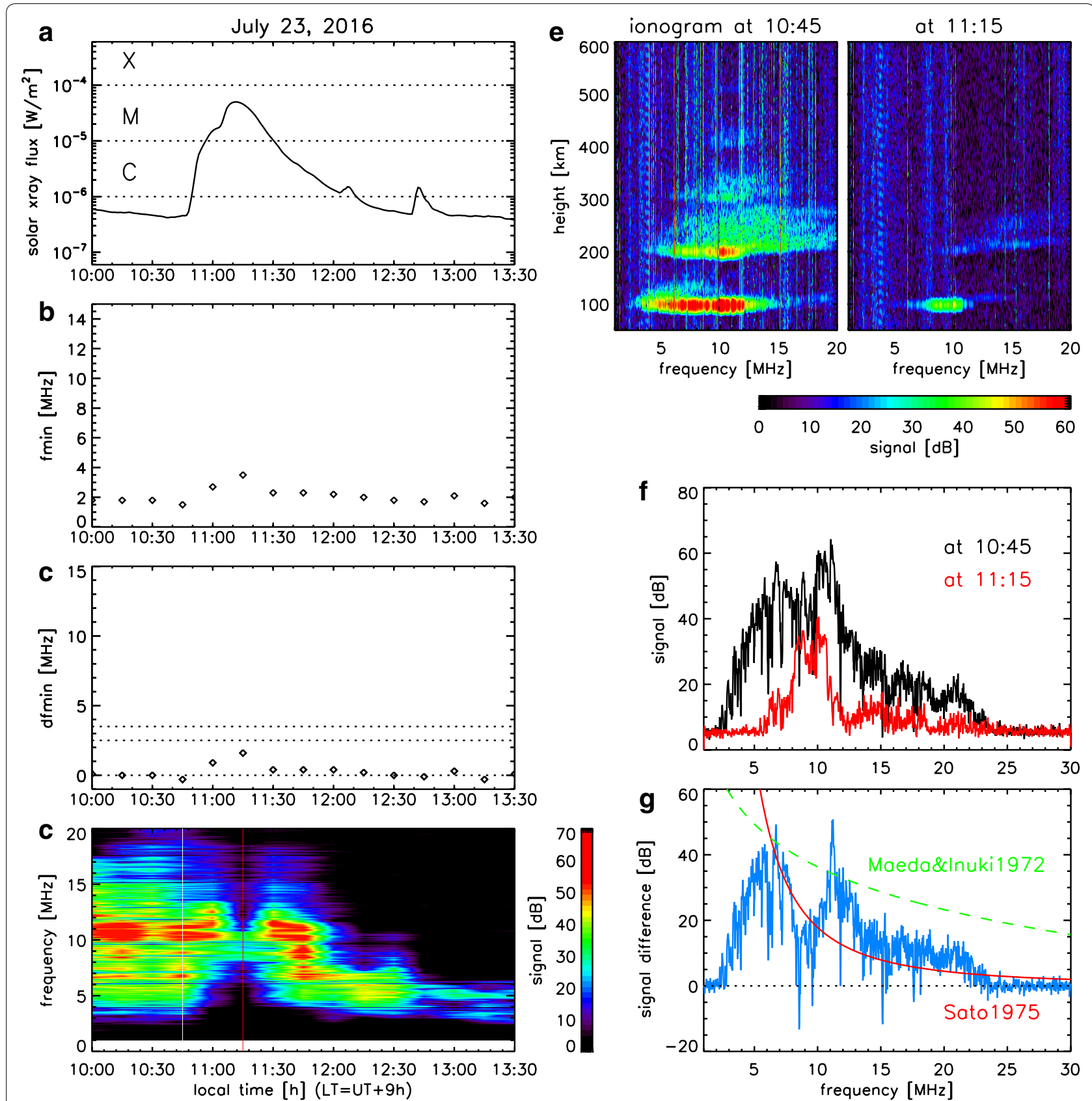

Fig. 8 Time variations in a solar X-ray flux, b fmin, c dfmin, and d maximum ionosonde signal at each frequency observed by VIPIR2 on July 23, 2016, e VIPIR2 ionograms at LT(=UT+ 9 h) 10:45 (left) and 11:15 (right), fVIPIR2 signal intensities before SWF at LT 10:45 (black) and during SWF at 11:15 (red), and g their difference representing SWF absorption (blue), as a function of frequency. Absorption intensities estimated using Eq. (10) from Maeda and Inuki (1972) and twice Eq. (2) from Sato (1975) are shown by the green dashed and red lines, respectively, in Fig. $8 \mathrm{~g}$ 
longitude)-Hamburg (53.50 $\mathrm{N}$ latitude, $9.96^{\circ} \mathrm{E}$ longitude), Hiraiso-Shepparton $\left(36.42^{\circ} \mathrm{S}\right.$ latitude, $145.51^{\circ} \mathrm{E}$ longitude), and Hiraiso-Lima (12.02 ${ }^{\circ} \mathrm{S}$ latitude, $77.1^{\circ} \mathrm{W}$ longitude) in March-June 1970 as follows:

$$
M=L+43.319 \log f-33.856 \cos \chi+3.037,
$$

where the effective solar zenith angle is represented by the smallest value among those at absorption points along the circuits. The absorption point, at an altitude of $90 \mathrm{~km}$, was estimated by assuming signal passes reflecting at the ground and at an altitude of $320 \mathrm{~km}$ (Maeda and Inuki 1972).

Maeda and Inuki (1972) found the following relationship between $M$ and the solar flux:

$$
M=0.996 \log F_{1}+15.365 \log F_{2}+9.673 \log F_{3}+82.585,
$$

where $F_{1}, F_{2}$, and $F_{3}$ represent the solar fluxes at the $0.5-3,1-8$, and $8-20 \AA$ bands, respectively, in units of $\mathrm{mW} / \mathrm{m}^{2}$. The solar flare class is usually determined by the maximum X-ray flux at $F_{2}$ during each flare event. Referring to the correlation between $F_{1}$ and $F_{2}$ and that between $F_{2}$ and $F_{3}$ from 1969 to 1970 shown by Maeda and Inuki (1972), we derive $F_{1}$ and $F_{3}$ as functions of $F_{2}$ as follows:

$$
\begin{aligned}
& F_{1}=0.11 F_{2}^{1.35} \\
& F_{3}=1.21 F_{2}^{0.75} .
\end{aligned}
$$

Using Eqs. (6)-(8), we obtain the relationship

$$
M=23.914 \log F_{2}\left(\mathrm{~mW} / \mathrm{m}^{2}\right)+82.431 .
$$

From Eqs. (5) and (9), we obtain

$$
L=23.914 \log F_{2}-43.319 \log f+33.856 \cos \chi+79.394 .
$$

The absorption intensity at $6.6 \mathrm{MHz}$ used for civil aviation communications as functions of solar flux and solar zenith angle derived from Eq. (10) is shown in Fig. 9a. A higher absorption intensity occurs for a larger solar flare and a smaller solar zenith angle. Figure 9c shows the dependence of absorption on frequency around the HF range. Absorption at lower frequencies is more effective for the same conditions, i.e., $90 \mathrm{~dB}$ at $1 \mathrm{MHz}, 35 \mathrm{~dB}$ at $6.6 \mathrm{MHz}$, and $30 \mathrm{~dB}$ at $30 \mathrm{MHz}$ for $\chi=0^{\circ}$ and $M=60$, roughly corresponding to an X2-class flare from Eq. (10).

\section{Comparison between observation and empirical relationships}

In this section, we compare the ionosonde observation and the empirical equations for absorption based on observations using a riometer (Eq. (2) from Sato (1975)) and circuit (Eq. (10) from Maeda and Inuki (1972)). As shown in "Absorption observed by VIPIR2" section, the small SWF signature at LT 11:15 on July 23, 2016, caused an absorption intensity of up to $40 \mathrm{~dB}$ at 5 and $12 \mathrm{MHz}$ at Kokubunji, Tokyo (35.71 ${ }^{\circ} \mathrm{N}$ latitude). Equation (2) for these observation conditions considering the round trip is also shown by the red curve in Fig. 8g. This function provides similar values at $6-10 \mathrm{MHz}$ and a similar decreasing trend at $12-22 \mathrm{MHz}$ to those in the observation, except for the enhanced absorption at $10-13 \mathrm{MHz}$ in the observation. This difference might be caused by the variation in signal reflection owing to the sporadic E-layer seen in the ionogram (Fig. 8e). No signals were observed at low frequencies $<6 \mathrm{MHz}$ owing to the large absorption, as expected from the equation. The relationship from Eq. (10) shown by the green dashed line was almost the upper limit of the observed absorption intensity. The discrepancy between the observation and the equation may be caused by the different signal passes: oblique propagation with more than three hops over a range from $8.1 \mathrm{Mm}\left(1 \mathrm{Mm}=10^{6} \mathrm{~m}\right)$ (for the Shepparton-Hiraiso circuit) to $15.4 \mathrm{Mm}$ (for the Lima-Hiraiso circuit) for Eq. (10), in contrast to vertical propagation with one round trip for this ionosonde observation and twice Eq. (2).

Next, we compare the dependences of these absorption equations on solar flux, solar zenith angle, and signal frequency. Figure $9 \mathrm{~b}$ shows the absorption intensity derived from Eq. (2) using the same color and format as those of Fig. 9a derived from Eq. (10). Equation (2) provides a much stronger frequency dependence, with absorption intensities of about 2000, 46, and $2.2 \mathrm{~dB}$ at frequencies of $1,6.6$, and $30 \mathrm{MHz}$, respectively, compared with those of 91, 55, and $27 \mathrm{~dB}$ from Eq. (10) for $X=0^{\circ}$ and X2-class flares (Fig. 9c).

Because the radio waves propagate over a long distance and are reflected by the ionosphere and ground multiple times, the oblique propagation of a long circuit passes through the ionosphere several times under various conditions (LT, latitude, and solar zenith angle) including the pass with the "effective solar zenith angle", which moderates the dependences on solar zenith angle and solar flux. According to a brief estimation of the oblique propagation in a spherical geometry, the incident angle for an $8 \mathrm{Mm}$ circuit with three hops is $\sim 7^{\circ}$ (Maeda and Inuki, 1972). The solar zenith angle of the absorbing ionospheric $D$ and $E$ regions varies in the circuit. Because the passing point of the $\mathrm{D}$ and $\mathrm{E}$ regions at the smallest solar zenith angle mostly affects the absorption, the oblique propagation increases the pass length by a factor of $1 / \sin \left(7^{\circ}\right) / 2 \approx$ 4 for the case of $8 \mathrm{Mm}$ distance compared with that for a vertical round trip. This results in the greater absorption 

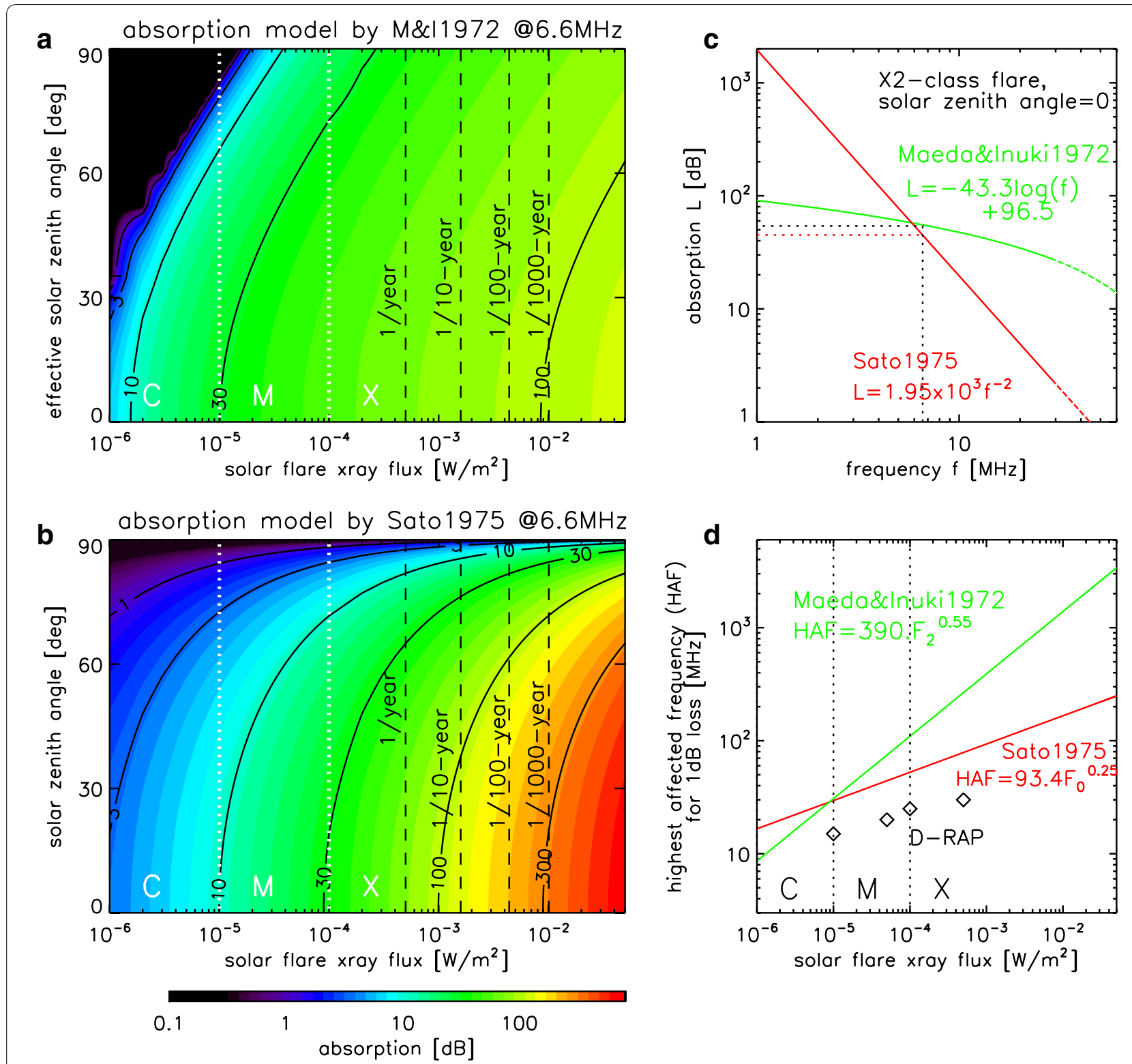

Fig. 9 Absorption intensity in $\mathrm{dB}$ at $6.6 \mathrm{MHz}$ as a function of solar flare $\mathrm{X}$-ray flux and (effective) solar zenith angle obtained using a Eq. (10) from Maeda and Inuki (1972) and b Eq. (2) from Sato (1975) with a common color contour shown by the color bar at the bottom, c absorption intensity for X2-class flare and solar zenith angle of $0^{\circ}$ as a function of signal frequency, and d highest affected frequency (HAF) as a function of solar flare X-ray flux. In C, d, the absorption intensity from Maeda and Inuki (1972) and that from Sato (1975) (considering the round trip for the HAF) are shown by green and red lines, respectively, and diamonds in $d$ are the HAFs used in D-RAP

of the oblique pass than that of the vertical pass. The SCNA observation is affected not only by the absorption in the ionospheric $\mathrm{D}$ and $\mathrm{E}$ regions but also by that in the F region. Mitra (1970) suggested that the contribution by the $\mathrm{F}$ region could even be dominant. The oblique propagation of a low-frequency signal is reflected at a low altitude in the $\mathrm{F}$ region and its absorption effect is expected to remain smaller than that in the vertical propagation (Eq. (2)) based on SCNA.

Figure 9d shows the highest affected frequency (HAF), which is defined as the highest frequency that experiences absorption of more than $1 \mathrm{~dB}$, as a function of solar X-ray flux estimated from twice Eq. (2) and from Eq. (10) as a function of solar X-ray flux for a solar zenith angle of $0^{\circ}$. Diamond marks show the HAF referred to the D-Region Absorption Prediction 
(D-RAP, https://www.swpc.noaa.gov/content/globa 1-d-region-absor ption-prediction-documentation). D-RAP is based on vertical round-trip propagation and considers the HF absorption caused by a solar flare and solar energetic particles. There is a difference in the HAF of a factor of two, but the dependence on the solar X-ray flux is almost the same as that in Eq. (2).

\section{Extreme event estimation for SWF absorption intensity}

For the absorption intensity in extreme cases, we use the solar flare occurrence with empirical Eqs. (2) and (10). The occurrence of flare classes shows a power-law distribution (e.g., Dennis, 1985). According to the results of a statistical analysis of solar flares by Gopalswamy (2018), the flare classes with occurrence probabilities of once per $1,10,100$, and 1000 years are X5.0, X15, X44, and X101, respectively. It has been reported that a solar-type star produces "superflares", which are two or more orders of magnitude larger than the largest flare observed on the Sun (Maehara et al. 2012). Although the possibility that superflares will occur in the current Sun is under discussion among experts, several studies predict the occurrence of a large solar flare, e.g., comparable energy to superflares (Shibata et al. 2013) or X75-X250 flare classes (Ishii et al. submitted to Earth Planets and Space) based on a physical viewpoint.

The corresponding scales are shown by vertical lines in Fig. 9a, b. For a $6.6 \mathrm{MHz}$ signal at a solar zenith angle of $0^{\circ}$, the once per $1,10,100$, and 1000 year absorption intensities from Eq. (10), respectively, become 71, 83, 93, and $100 \mathrm{~dB}$ and those from Eq. (2) become 71, 130, 210 , and $320 \mathrm{~dB}$ for these probabilities, as summarized in Table 3.

The degree of ionization of the upper atmosphere is usually on the order of $10^{-6}$, so increasing the solar X-ray flux by a factor of $10-100$ places it in the range, where similar ionization processes are expected. Therefore, this extrapolation is considered reasonable.

\section{Summary}

Using long-term (36 years) ionosonde data observed by NICT at Kokubunji, Tokyo, with proposed empirical equations based on ionosonde, SCNA, and HF circuit observations, we investigated SWF absorption and duration as follows.

1. Preceding the analysis, we examined the dependences of fmin on solar X-ray flux and solar zenith angle. As in previous studies, fmin increases with the solar flux and angle. The parameter $\mathrm{d}$ fmin, which is fmin subtracted by its 27-day running median at the same LT, shows a dependence on solar X-ray flux and solar zenith angle that is much closer to that described by the empirical relationship from Sato (1975) than fmin if the background fmin is taken into account.

2. We obtained the SWF duration separately for three criteria: $\mathrm{d} f \min \geq 2.5 \mathrm{MHz}, \mathrm{d} f \min \geq 3.5 \mathrm{MHz}$, and blackout. The duration is up to $8 \mathrm{~h} 15 \mathrm{~min}$ for $\mathrm{d}$ fmin $\geq 2.5 \mathrm{MHz}$, up to $5 \mathrm{~h} 15 \mathrm{~min}$ for $\mathrm{d} f \mathrm{~min} \geq 3.5 \mathrm{MHz}$, and up to $3 \mathrm{~h} 15 \mathrm{~min}$ for blackout, with decreasing occurrence with increasing duration. The duration distributions are similar among the different SWF criteria. The duration increases with the peak X-ray flux associated with solar flares. The occurrence of events also depends on the local time, i.e., solar zenith angle.

3. The observed signal absorption estimated from a small SWF signature on July 23, 2016, matches well with the empirical relationship proposed by Sato (1975) over most of the frequency range. We obtained a generalized empirical relationship for absorption from the long-distance multihop circuit observation proposed by Maeda and Inuki (1972) as a function of solar X-ray flux of 1-8 $\AA$. The dependences of the latter relationship on solar flare X-ray flux, solar zenith angle, and signal frequency are more moderate than those obtained from the vertical observation.

4. The absorption and duration of extreme SWF events with occurrence probabilities of once per 1, 10, 100, and 1000 years were estimated from the analysis results. The absorption intensity at $6.6 \mathrm{MHz}$ at a solar zenith angle of $0^{\circ}$ becomes $71-100 \mathrm{~dB}$ for oblique propagation and $71-320 \mathrm{~dB}$ for a vertical round-trip pass, and the estimated duration reaches $7.4 \mathrm{~h}$ for blackout and up to $12 \mathrm{~h}$ for the $\mathrm{d} f \mathrm{~min} \geq 2.5 \mathrm{MHz}$ criterion.

Table 3 Estimated absorbtion intensities for extreme events

\begin{tabular}{llllll}
\hline Absorbtion intensity $(\mathbf{d B})$ & Once per year & Once per $\mathbf{1 0}$ years & Once per $\mathbf{1 0 0}$ years & Once per 1000 years & References \\
\hline Long circuit $\mathrm{X}=0$ and $\mathrm{f}=6.6 \mathrm{MHz}$ & 71 & 83 & 93 & 100 & Equation (10) Maeda \\
and Inuki (1972) & & 320 & Equation (2) Sato (1975) \\
Vertical $X=0$ and $\mathrm{f}=6.6 \mathrm{MHz}$ & 71 & 130 & 210 &
\end{tabular}


Our analysis provides quantitative values of the SWF absorption and duration for different $\mathrm{d}$ fmin thresholds. For the quantitative evaluation of the absorption intensity, further study with more events using current and future VIPIR2 observations is required. In addition, comparison with SWF absorption intensity observed with a riometer and SuperDARN would be useful (e.g., Fiori et al. 2018). The occurrence probabilities of extreme SWF events are provided by this study for the first time. The duration of SWF events is useful information for the operation of radio communication systems to predict the recovery time and to prepare alternative means of communication during the events. This information is also expected to contribute to designing operation systems with sufficient resistance to space weather disasters.

\section{Abbreviations}

D-RAP: D-region absorption prediction; FWHM: Full width of half maximum; GNSS: Global Navigation Satellite System; GOES: Geostationary Operational Environmental Satellite; HAF: Highest affected frequency; HF: High-frequency; LT: local time; NICT: National Institute of Information and Communications Technology; NOAA: National Oceanic and Atmospheric Administration; SCNA: Sudden cosmic noise absorption; SID: Sudden ionospheric disturbance; SITEC: Sudden increase in total electron content; SWF: Short-wave fadeout; VIPIR2: Vertical Incidence Pulsed lonospheric Radar 2

\section{Acknowledgements}

CT thanks Dr. K. Nozaki, Dr. T. Maruyama, Dr. H. Maeno, and Dr. I. Yamazaki for useful discussions.

\section{Authors' contributions}

CT conducted the research and is responsible for the results presented in this paper. MN is responsible for the observation data set and supported this analysis. SS, TT, and MI contributed to the discussion as experts on the ionosphere and its social impacts. DS, KW, and NN contributed to the discussion as experts on solar physics. All authors read and approved the final manuscript.

\section{Funding}

This work was supported by MEXT/JSPS KAKENHI Grants $15 \mathrm{H} 05813$ and 19K03942.

\section{Availability of data and materials}

The original ionogram data set is available from the ionogram viewers at http://wdc.nict.go.jp/IONO/index_E.html. Manually scaled ionosonde parameters of $1 \mathrm{~h}$ resolution are archived at http://wdc.nict.go.jp/IONO/HP2009/ISDJ/ manual_txt.html, and the data set of 15 min resolution will be provided to the scientific community on request (see "contact us" on the access tab of http:// wdc.nict.go.jp/IONO/index_E.html). We used the Geostationary Operational Environmental Satellite (GOES) flare list provided by NOAA National Centers for Environmental Information at https://www.ngdc.noaa.gov/stp/space-weath er/solar-data/solar-features/solar-flares/x-rays/goes/xrs/.

\section{Ethics approval and consent to participate}

Not applicable.

\section{Consent for publication}

Not applicable.

\section{Competing interests}

The authors declare that they have no competing interests.

\section{Author details}

${ }^{1}$ National Institute of Information and Communications Technology (NICT), Tokyo, Japan. ${ }^{2}$ Electronic Navigation Research Institute (ENRI), National
Institute of Maritime, Port and Aviation Technology (MPAT), Tokyo, Japan.

${ }^{3}$ National Defense Academy of Japan, Kanagawa, Japan.

Received: 22 May 2020 Accepted: 21 September 2020

Published online: 13 November 2020

\section{References}

Aulanier G, Démoulin P, Schrijver CJ, Janvier M, Pariat E, Schmieder B (2013) The standard flare model in three dimensions. II. Upper limit on solar flare energy. Astron Astrophys. 549:66

Barta V, Sátori G, Berényi KA, Kis Á, Williams E (2019) Effects of solar flares on the ionosphere as shown by the dynamics of ionograms recorded in Europe and South Africa. Ann Geophys 37:747-761. https://doi.org/10.5194/angeo $-37-747-2019$

Chakraborty S, Ruohoniemi JM, Baker JBH, Nishitani N (2018) Characterization of short-wave fadeout seen in daytime SuperDARN ground scatter observations. Radio Sci 53:472-484. https://doi.org/10.1002/2017RS006488

Chakraborty S, Baker JBH, Ruohoniemi JM, Kunduri BSR, Nishitani N, Shepherd SG (2019) A study of SuperDARN response to co-occurring space weather phenomena. Space Weather 17:1351-1363. https://doi.org/10.1029/2019S W002179

Davies K (1996) Sudden ionospheric disturbances. In: Dieminger W, Hartmann GK, Leitinger R (eds) The upper atmosphere: data analysis and interpretation. Springer, Berlin, pp 706-722

Dellinger JH (1937) Sudden disturbances of the ionosphere. J Appl Phys 8:732-751

Dennis BR (1985) Solar hard X-ray bursts. Solar Phys. 100:465-490

Deshpande SD, Subrahmanyam CV, Mitra AP (1972) lonospheric effects of solar flares-I. The statistical relationship between X-ray flares and SIDs. J Atmos Terr Phys 34:211-227

Fiori RAD, Koustov AV, Chakraborty S, Ruohoniemi JM, Danskin DW, Boteler DH, Shepherd SG (2018) Examining the potential of the Super Dual Auroral Radar Network for monitoring the space weather impact of solar X-ray flares. Space Weather 16:1348-1362. https://doi.org/10.1029/2018SW0019 05

Fletcher L, Dennis BR, Hudson HS, Krucker S, Phillips K, Veronig A, Battaglia M, Bone L, Caspi A, Chen Q, Gallagher P, Grigis PT, Ji H, Liu W, Milligan RO, Temmer M (2011) An observational overview of solar flares. Space Sci Rev. 159:19-106. https://doi.org/10.1007/s11214-010-9701-8

Gopalswamy N (2018) Extreme solar eruptions and their space weather consequences. In: Buzulukova N (ed) Extreme events in Geospace origins, Predictability, and consequences. Elsevier, Amsterdam, pp 37-63. https:// doi.org/10.1016/C2016-0-03769-5

Handzo R, Forbes JM, Reinisch B (2014) lonospheric electron density response to solar flares as viewed by digisondes. Space Weather 12:205-216. https://doi. org/10.1002/2013SW001020

Hendl RG, Skrivanek RA (1973) Statistical analysis of shortwave fadeout occurrence for the years 1955 to 1969, Environ. Res. Papers, 452, AFCRL-TR-73-0385

Maeda R, Inuki H (1972) Magnitude of short-wave fade-out. J Radio Res Lab 18(99):467-476

Maehara H, Shibayama T, Notsu S, Notsu Y, Nagao T, Kusada S, Honda S, Nogami D, Shibata K (2012) Superflares on solar-type stars. Nature 485:478-481. https://doi.org/10.1038/nature11063

Mitra AP (1970) HF and VHF absorption techniques in radio wave probing of the ionosphere. J Atmos Terr Phys 32:623-646

Nishitani N et al (2019) Review of the accomplishments of mid-latitude Super Dual Auroral Radar Network (SuperDARN) HF radars. Prog Earth Planet Sci 6:27. https://doi.org/10.1186/s40645-019-0270-5

Reep JW, Knizhnik KJ (2019) What determines the X-ray intensity and duration of a solar flare? Astrophys J 874(157):1-16

Riley P (2012) On the probability of occurrence of extreme space weather events. Space Weather 10:S02012. https://doi.org/10.1029/2011SW000734

Sato T (1975) Sudden fmin enhancements and sudden cosmic noise absorptions associated with solar X-ray flares. J Geomagn Geoelect 27:95-112

Shibata K, Isobe H, Hillier A, Choudhuri AR, Maehara H, Ishii TT, Shibayama T, Notsu S, Notsu Y, Nagao T, Honda S, Nogami D (2013) Can superflares occur on our Sun? Publ Astron Soc Jpn 65(49):1-8 
US National Science and Technology Council (2018) Space Weather Phase 1 Benchmarks, Available via DIALOG https://www.whitehouse.gov/wp-conte nt/uploads/2018/06/Space-Weather-Phase-1-Benchmarks-Report.pdf

\section{Publisher's Note}

Springer Nature remains neutral with regard to jurisdictional claims in published maps and institutional affiliations.
Submit your manuscript to a SpringerOpen ${ }^{\odot}$ journal and benefit from:

- Convenient online submission

- Rigorous peer review

- Open access: articles freely available online

- High visibility within the field

Retaining the copyright to your article

Submit your next manuscript at $\boldsymbol{\nabla}$ springeropen.com 